\title{
DESY AND ILC EDMS: ENGINEERING DATA MANAGEMENT FOR LARGE SCIENTIFIC PROJECTS
}

\author{
J. Bürger, J. A. Dammann, S. Eucker, L. Hagge* , A. Herz, J. Kreutzkamp, S. Pantó, D. Szepielak, \\ P. Tumidajewicz, N. Welle, DESY, Hamburg, Germany
}

\section{Abstract}

DESY is using an Engineering Data Management System (EDMS) for supporting the XFEL and PETRA III projects by e.g. enabling 3D CAD design collaboration, managing planning documents, supporting the manufacturing processes of components and providing general project documentation. The EDMS is currently adopted by the ILC global design effort for the preparation of the Engineering Design Report.

The DESY and ILC EDMS is based on a commercial system which has been customized to the specific needs of large scientific projects. They include, in addition to general engineering requirements, the support of ad-hoc teamwork and powerful yet easy-to-use web frontends. The paper gives an overview of the EDMS capabilities and describes experience and benefits of using the EDMS.

\section{INTRODUCTION}

DESY has introduced an Engineering Data Management System (EDMS) for managing the complexity and optimizing the design and production processes in its next-generation accelerator projects. Applications of the DESY EDMS include:

- Reviewing and approving the construction drawings of the new PETRA III experimental hall;

- Planning the XFEL buildings, utilities and technical infrastructure [1];

- Supporting the manufacturing and preparations processes of superconducting cavities for TTF and XFEL [2];

- 3D CAD collaboration of DESY and FNAL in the common development of an accelerator module for the TTF.

The DESY EDMS has been extended for the needs of

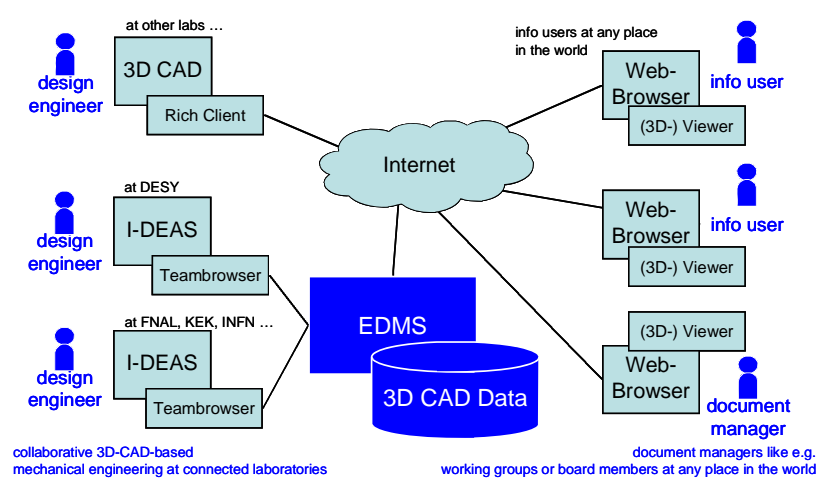

Figure 1: Overview of the EDMS architecture. The EDMS is fully web-based. the ILC collaboration and in that configuration is labelled ILC EDMS. The EDMS is fully web based and has its strengths in its capabilities for 3D CAD design collaboration [3] with generally-available viewing, its strong web interfaces, its workflow capabilities and its support for team work. Figure 1 illustrates architecture of the DESY and ILC EDMS.

\section{WHAT IS AN EDMS?}

An EDMS is an information system framework which contains functionality for document management, 3D CAD Data Management, configuration and version control, workflow automation and many other management disciplines.

Before an EDMS can be used, it has to be configured for its project by defining document types and relation types, implementing workflows for reviewing, approval and change activities, configuring navigational and organizational structures, providing interfaces to external applications, and many other issues. Configuring an EDMS is usually an ongoing effort throughout the project lifetime, as novel requirements are continuously emerging as the project progresses.

\section{EDMS OBJECTIVES}

The EDMS is the central collaboration and lifecycle management platform in an accelerator project. The EDMS objectives include:

- Enable collaboration members to access and contribute project information over the web;

- Enable engineers at different laboratories to collaboratively design components using 3D CAD;

- Enable scientists to participate in design processes from the very beginning by viewing and red-lining the evolving CAD models;

- Provide teams, committees, boards etc. with workspaces for work-in-progress document management;

- Support change control of critical documents and of the accelerator baseline configurations;

- Enable component tracking during manufacturing, installation and maintenance procedures;

- Protect confidential information and intellectual property against unauthorized access

\section{BASIC CONCEPTS}

The EDMS can handle any item which is available as an electronic document, i.e. a file consisting of texts, graphics, figures and (binary) data, as for example reports, notes, minutes, drawings, 3D models, 

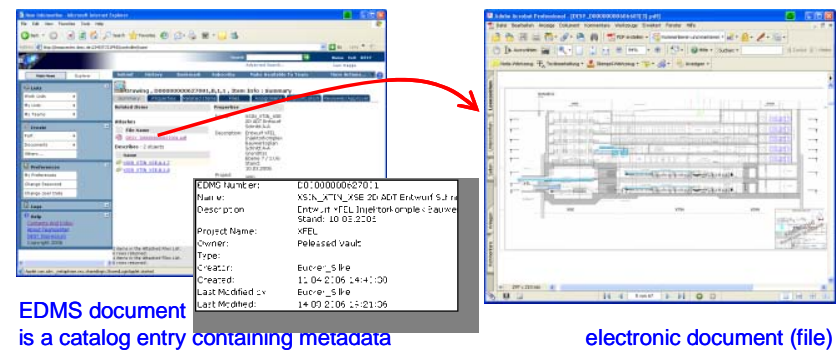

Figure 2: EDMS items are catalog entries which contain metadata and point to electronic files.

simulations, multimedia, contracts and many more. The EDMS differentiates between documents (or more generally, items), which are index cards with metadata, and electronic documents, which correspond to the accessible file. Items can be represented by several files in different formats. Figure 2 illustrates the relation of documents and electronic documents (file). Figure 3 shows a screenshot of the EDMS web interface displaying a list of items with color-coded item states.

Figure 4 illustrates the logical workspaces within the EDMS. Teams are work-in-progress areas for workgroups, while the projects vault contains projectspecific areas for the released and approved items of the different projects. The EDMS scales from workgroup to worldwide collaboration.

\section{EDMS CAPABILITIES}

The following sections highlight some selected EDMS capabilities.

\section{Team Workspaces}

Teams are private work-in-progress areas which behave like shared web file spaces. They are selfadministrated units with local folder structures. Teams are intended for use by work groups, boards and committees, and for temporary constellations like e.g. during tendering procedures or for collaboration with industrial partners.

\section{Project Repositories}

The projects vault contains items which have been

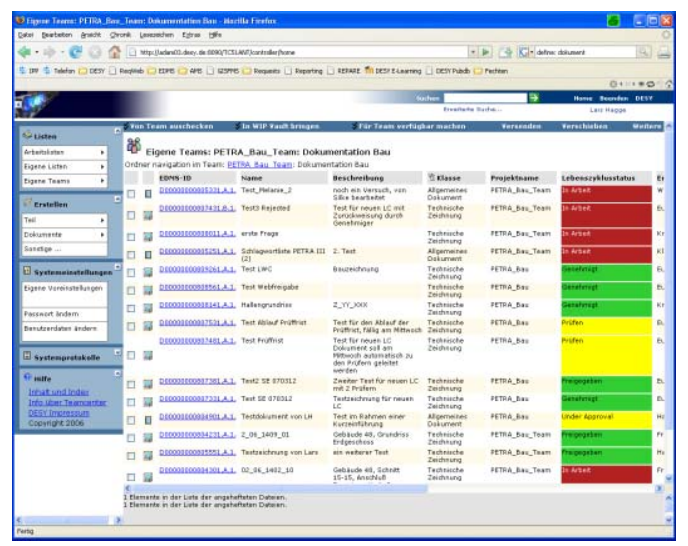

Figure 3: Item listing in EDMS web interface showing item name, description, status, type and author.

released or approved by their teams, and it implements the projects' access control policies. The projects vault behaves like a web-like information repository which is accessed by a powerful search engine and allows creating hyperlink-like relations between items.

\section{Life Cycles and Assignments}

A workflow engine routes items between users for processing. Items are promoted according to predefined processes, and users are notified by e-mail whenever they receive new assignments. Workflows are used for coordination tasks like notifications, reviews and approvals, and this way move items between team and project workspaces. They can also be used to implement more sophisticated processes: For example, in the preparation of s.c. cavities, the workflow engine is also used to route work instructions to process teams and receive their resulting inspection sheets [2].

\section{D CAD Data Management}

The EDMS seamlessly integrates document management and 3D CAD data management, as it treats parts and CAD models and drawings in the same way as documents wrt e.g. storage, navigation and retrieval. Parts and CAD models contain additional relations and hierarchies, esp. the bill of material (BOM), which lists

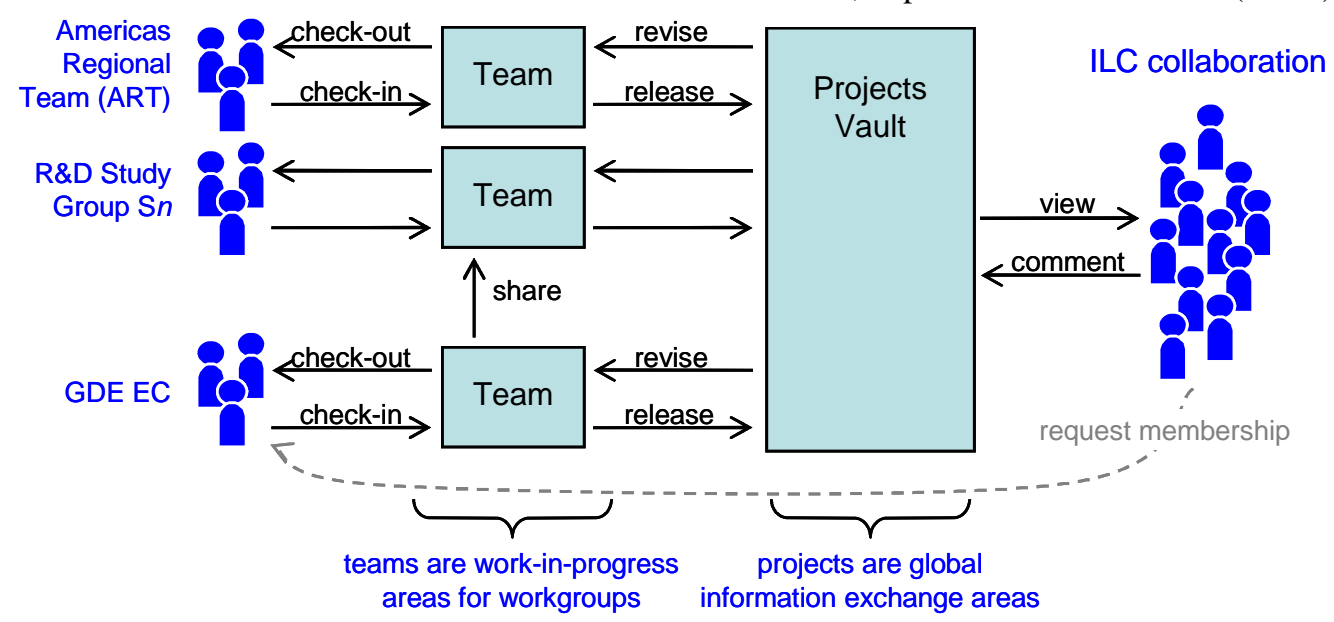

Figure 4 Basic EDMS operation principle with examples from the ILC. 
all the subsystems and components which are used in an assembly. Figure 5 shows the item details of an accelerator module and its BOM as they are displayed in the EDMS web interface.

Defining and standardizing the system breakdown structure (i.e. the accelerator subsystems, components etc.) and organizing workflows and data management around this hierarchy is one of the essential concepts of engineering data management.

\section{Component Tracking}

Components are manufactured according to CAD models. The EDMS treats these components as so-called serialized parts and is able to track their manufacturing and installation processes and relate them with product documentation, inspection sheets and other documentation.

Figure 6 shows an example for the manufacturing documentation of cavities. The CAD BOM states that a cavity contains eight dumb-bells. The serialized cavity contains eight distinct dumb-bells which are identified by their serial numbers. The EDMS tracks for each serialized part from which CAD assembly it has been created.

\section{INTEGRATING EDMS}

Letting collaboration partners contribute their ideas and experience and involve their experts, methods, tools implies that the EDMS has to accept partner environments as much as possible as they are and provide open integration mechanisms for (m)any external applications. The DESY and ILC EDMS offers a set of
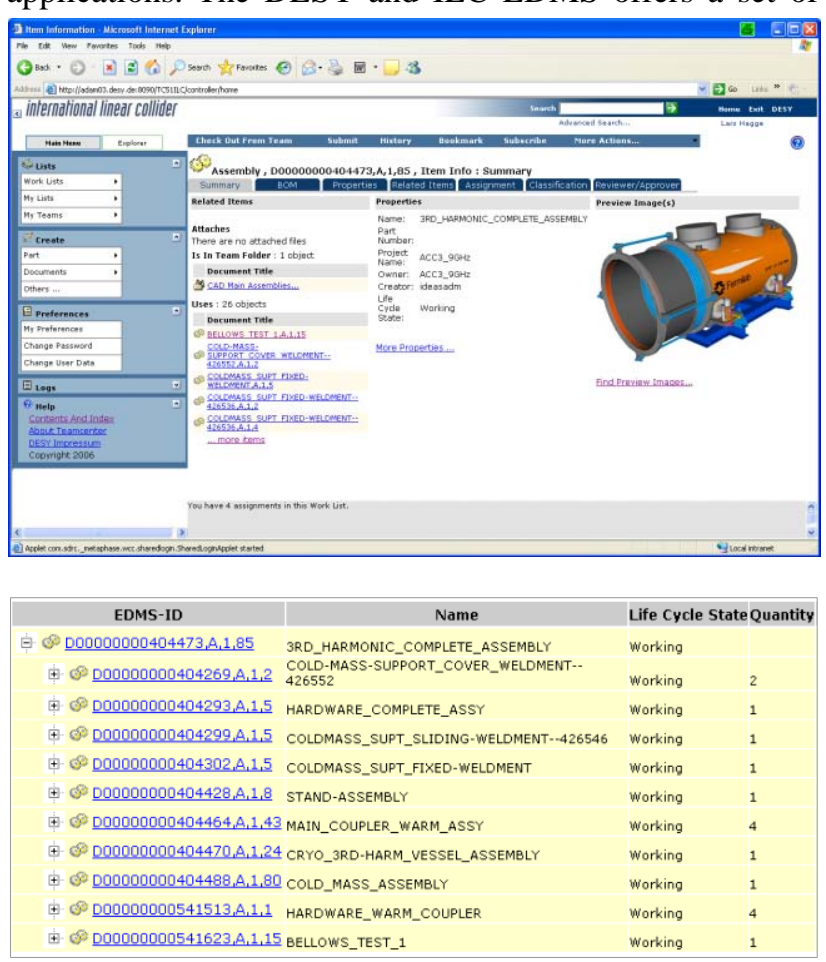

Figure 5: 3D CAD Data in the EDMS: Item details (top) and bill of material (product breakdown structure, bottom).

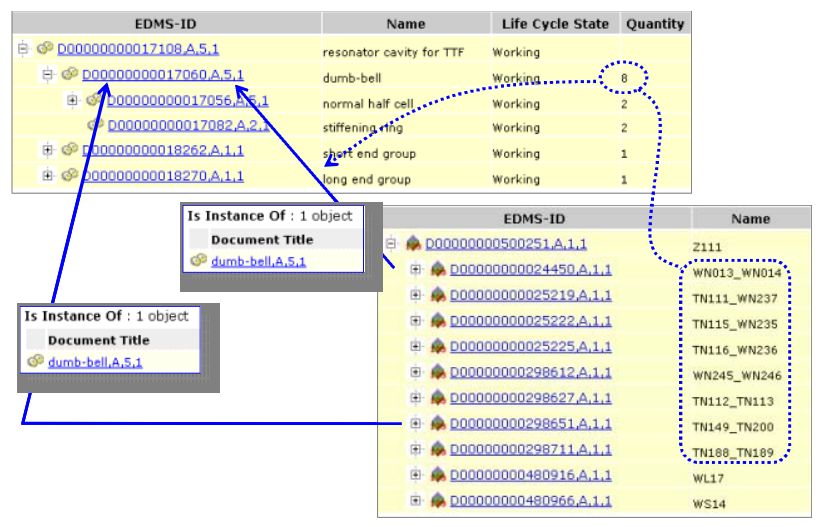

Figure 6: Example for component tracking in manufacturing processes (see text).

generic REST-based web services which can be used to exchange information with external systems, build applications on top of EDMS, or directly access EDMS items from personal Web pages [4].

\section{EXPERIENCE}

In accelerator projects, many of the engineering issues are technically the same as elsewhere, but the complexity and distribution especially in the global 3D CAD design collaboration is currently at the leading edge of technology and hardly maintainable without an EDMS.

The EDMS promotes a staged approach to exposing complexity, which proposes shared workspaces and common tools and procedures within workgroups, and web-ish connections and interactions between different teams.

One crucial challenge which can be overcome with EDMS is the early integration of scientists into mechanical engineering design processes, as the EDMS enables scientists to view CAD data and provide feedback by e.g. commenting and redlining.

As most of the EDMS users are casual users, acceptance and making the system intuitively usable in the user's world are the key issue for a successful EDMS deployment.

\section{REFERENCES}

[1] J. Bürger et al., "Establishing a Collaborative Planning Procedure for the European XFEL", PAC 2005, Knoxville, May 2005.

[2] J. Bürger et al., "Towards Industrialization: Supporting the Manufacturing Processes of Superconducting Cavities at DESY”, Phys C, 441(2006)268-271

[3] D. A. Venton, "Planning for ILC Success Through Shared Databases", ILC NewLine, online at http://www.linearcollider.org/newsline/archive/2006/ 20061214.html

[4] D. Szepielak, "REST-based Service Oriented Architecture for Dynamically Integrated Information Systems”, PhD Symposium at ICSOC 2006, Chicago, December 2006. 